\title{
Riqueza fitoplanctónica en el lago Zempoala del Parque Nacional Lagunas de Zempoala
}

\author{
Phytoplankton wealth in Zempoala lake of Lagunas de Zempoala National Park
}

\author{
Migdalia Díaz Vargas \\ ORCID: 0000-0002-0510-8358/migdalia@uaem.mx \\ Profesora-investigadora, Laboratorio de Hidrobilogía, Centro de Investigaciones Biológicas (CIB), Universidad Autónoma del Estado de Morelos (UAEM)
}

\section{Héctor Eduardo Franco Cotero}

ORCID: 0000-0002-8054-7078/francoshark23@gmail.com

Licenciatura en Biología, Facultad de Ciencias Biológicas (FCB), UAEM

\section{Judith García Rodríguez}

ORCID: 0000-0003-3789-1938/garciarj@uaem.mx

Profesora-investigadora, Laboratorio de Hidrobiología, CIB, UAEM

\section{Elsah Arce Uribe}

ORCID: 0000-0002-9815-2525/elsah.arce@uaem.mx

Profesora-investigadora, Laboratorio de Acuicultura, CIB, UAEM

\section{RESUMEN}

Este estudio examina la riqueza de especies fitoplanctónicas que se encuentran en uno de los cuerpos acuíferos del Parque Nacional Lagunas de Zempoala. Los muestreos fueron realizados mensualmente entre septiembre de 2018 a febrero de 2019. Durante este periodo, se reconocieron 26 especies integradas en siete divisiones, 20 familias y siete clases, dentro de las que destaca la clase Bacillariophyceae con la mayor diversidad (ocho especies). La especie Staurastrum bulbosum fue la más frecuente durante todo el estudio. En cuanto a la diversidad temporal, octubre presentó 15 especies y septiembre 13. Estas variaciones tienen que ver con cambios en las condiciones abióticas a lo largo del año. De acuerdo con trabajos anteriores, el lago Zempoala puede clasificarse como mesotrófico con periodos de eutrofia relacionados principalmente con la disponibilidad de nutrientes y los florecimientos algales.

PALABRAS CLAVE

fitoplancton, riqueza de especies, lago de Zempoala
ABSTRACT

This study examines the wealth of phytoplanktonic species found in one of the aquifers of the Lagunas de Zempoala National Park. The samples were carried out monthly from September 2018 to February 2019. During this period, 26 species integrated in seven divisions, 20 families and seven classes were recognized, among which the Bacillariophyceae class stands out with the greatest diversity (eight species). The species Staurastrum bulbosum was the most frequent throughout the study. In terms of temporal diversity, October presented 15 species and September 13, these variations have to do with changes in abiotic conditions throughout the year. According to previous work, the lake Zempoala can be classified as mesotrophic with periods of eutrophy mainly related to the availability of nutrients and algal blossoming.

KEY WORDS

phytoplankton, species wealth, Zempoala lake, aquifers 
El fitoplancton está conformado por todos aquellos microorganismos fotosintetizadores que se encuentran suspendidos (flotando o nadando) en la columna de agua de ríos, lagos y océanos (E. Graham et al., 2016; G. Wetzel, 2001). Estos microorganismos constituyen una parte fundamental del ecosistema donde se desarrollan, ya que son productores primarios de la cadena trófica; además, el fitoplancton contribuye en gran medida en la producción de oxígeno atmosférico, aportando cerca de la mitad de éste (Mora Navarro et al., 2004), y se encuentra estrechamente involucrado en los ciclos biogeoquímicos, como los ciclos del carbono, nitrógeno, sílice y fósforo (E. Graham et al., 2016).

El conocimiento que se tiene del fitoplancton en México ha crecido a un ritmo lento y pausado. En el caso del Parque Nacional Lagunas de Zempoala, Rodríguez y Tavera (1998) registraron 27 especies. En años posteriores se publicó un registro de 29 especies, siendo la clase Bacillariophyceae el grupo más diverso y abundante (Rodríguez et al., 2003; Rodríguez et al., 2010). En trabajos más recientes se estudió la diversidad algal de tres lagos del Parque Nacional Lagunas de Zempoala, incluyendo especies fitoplanctónicas, metaplanctónicas, bentónicas y epifitas, y sin considerar a la clase Bacillariophyceae. Como resultado se obtuvo un total de 55 especies, de las cuales predominaron los grupos Chlorophyta y Cyanophyta (Godínez Ortega et al., 2017). El presente estudio tiene como objetivo contribuir al conocimiento de la diversidad de especies fitoplanctónicas presentes en el lago Zempoala.

\section{Materiales y métodos}

El lago Zempoala se ubica en el Parque Nacional Lagunas de Zempoala que, a su vez, se localiza en las coordenadas $19^{\circ} 06^{\prime}$ oo" latitud norte y $99^{\circ} 16^{\prime}$ oo" longitud oeste, y abarca 4,790 hectáreas de los municipios de Ocuilán, estado de México, y Huitzilac, Morelos, México. La recolecta del material biológico para este estudio se realizó a lo largo de un transecto de la zona litoral a la zona pelágica del lago utilizando una embarcación con pedales. Los muestreos fueron realizados mensualmente, de septiembre a diciembre de 2018 y en febrero de 2019.

La recolecta de las muestras se efectuó con una red de plancton de abertura de poro de $60 \mu \mathrm{m}$. El material biológico fue depositado en recipientes plásticos y se preservó con formol al 4\%. El reconocimiento de las especies se realizó utilizando un microscopio compuesto Leica DM500, con módulo de cámara integrado ICC50 para la toma de fotografías, así como empleando trabajos especializados y una base de datos electrónica para la ubicación taxonómica de las especies (Godínez Ortega et al., 2017; Guiry y Guiry, 2020; Ortega, 1984).

\section{Resultados}

De las especies de fitoplancton, 26 fueron reconocidas e integradas en siete clases, veinte familias y siete divisiones: Bacillariophyta, Charophyta, Cryptophyta, Chlorophyta, Cyanobacteria, Miozoa y Euglenozoa (tabla 1). La clase Bacillariophyceae fue la más diversa, con ocho 
especies, seguida de las clases Charophyceae y Chlorophyceae, con cinco especies cada una; para Cyanophyceae se reconocieron cuatro especies y Dinophyceae presentó dos especies, mientras que Euglenophyceae y Cryptophyceae contaron con una especie cada una (figuras 1 a 4).

En términos de porcentaje, Bacillariophyceae representa el 31\% del total de especies observadas a lo largo de los cinco meses de muestreo; Charophyceae y Chlorophyceae representan cada una el 19\% del total de especies; Cyanophyceae el 15\%; Dinophyceae el 8\%, y Euglenophyceae y Cryptophyceae el $4 \%$ cada una.

Tabla 1

Listado taxonómico de las especies reconocidas durante los meses de colecta

\begin{tabular}{|c|c|c|c|c|c|}
\hline TAXA & $\begin{array}{l}\text { SEPTIEMBRE } \\
2018\end{array}$ & $\begin{array}{l}\text { OCTUBRE } \\
2018\end{array}$ & $\begin{array}{l}\text { NOVIEMBRE } \\
2018\end{array}$ & $\begin{array}{l}\text { DICIEMBRE } \\
2018\end{array}$ & $\begin{array}{l}\text { FEBRERO } \\
2019\end{array}$ \\
\hline \multicolumn{6}{|l|}{ 1. Bacillariophyta } \\
\hline $\begin{array}{l}\text { Clase Bacillariophyceae } \\
\text { Familia Cocconeidaceae }\end{array}$ & & & $x$ & & \\
\hline \multicolumn{6}{|l|}{$\begin{array}{l}\text { Cocconeis placentula } \\
\text { Familia Cymbellaceae }\end{array}$} \\
\hline $\begin{array}{l}\text { Cymbella sp. } \\
\text { Familia Fragilariaceae }\end{array}$ & & $x$ & & & \\
\hline $\begin{array}{l}\text { Fragilaria crotonensis } \\
\qquad \text { Familia Gomphonemataceae }\end{array}$ & $\mathrm{x}$ & $\mathrm{x}$ & & $x$ & \\
\hline $\begin{array}{l}\text { Gomphonema acuminatum } \\
\text { Familia Rhopalodiaceae }\end{array}$ & & $x$ & & & \\
\hline Epithemia turgida & & $x$ & & $x$ & $x$ \\
\hline Ephitemia sorex & & $x$ & & $x$ & \\
\hline $\begin{array}{l}\text { Rhopalodia sp. } \\
\text { Familia Tabellariaceae }\end{array}$ & & $x$ & & & $x$ \\
\hline Asterionella Formosa & $x$ & $x$ & & & $x$ \\
\hline \multicolumn{6}{|l|}{ 2. Charophyta } \\
\hline \multicolumn{6}{|l|}{$\begin{array}{c}\text { Clase Zygnematophyceae } \\
\text { Familia Closteriaceae }\end{array}$} \\
\hline $\begin{array}{l}\text { Closterium acutum } \\
\text { Familia Desmidiaceae }\end{array}$ & & & & $x$ & \\
\hline Cosmarium subtumidum & $x$ & & & & \\
\hline $\begin{array}{l}\text { Staurastrum bulbosum } \\
\text { Familia Zygnemataceae }\end{array}$ & $x$ & $x$ & $x$ & $x$ & $x$ \\
\hline Mougeotia sp. & $x$ & & & $x$ & \\
\hline Zygnema sp. & & & & & $x$ \\
\hline \multicolumn{6}{|l|}{ 3. Cryptophyta } \\
\hline $\begin{array}{l}\text { Clase Cryptophyceae } \\
\quad \text { Familia Cryptomonadaceae }\end{array}$ & & & & & \\
\hline
\end{tabular}

Año 16, núm. 40, noviembre 2020-marzo 2021 


\begin{tabular}{|c|c|c|c|c|c|}
\hline TAXA & $\begin{array}{l}\text { SEPTIEMBRE } \\
2018\end{array}$ & $\begin{array}{l}\text { OCTUBRE } \\
2018\end{array}$ & $\begin{array}{l}\text { NOVIEMBRE } \\
2018\end{array}$ & $\begin{array}{l}\text { DICIEMBRE } \\
2018\end{array}$ & $\begin{array}{l}\text { FEBRERO } \\
2019\end{array}$ \\
\hline Cryptomonas ovata & $x$ & & & & \\
\hline \multicolumn{6}{|l|}{ 4. Chlorophyta } \\
\hline \multicolumn{6}{|l|}{$\begin{array}{l}\text { Clase Chlorophyceae } \\
\text { Familia Scenedesmaceae }\end{array}$} \\
\hline $\begin{array}{l}\text { Scenedesmus quadricauda } \\
\text { Familia Hydrodictyaceae }\end{array}$ & & $x$ & & & \\
\hline $\begin{array}{l}\text { Stauridium tetras } \\
\quad \text { Familia Sphaeropleaceae }\end{array}$ & $\mathrm{x}$ & & & & \\
\hline $\begin{array}{r}\text { Radiofilum conjunctivum } \\
\text { Familia Volvocaceae }\end{array}$ & $\mathrm{x}$ & & & & \\
\hline Eudorina elegans & & $x$ & $x$ & $x$ & \\
\hline Volvox sp. & & & & & $x$ \\
\hline \multicolumn{6}{|l|}{ 5. Cyanobacteria } \\
\hline \multicolumn{6}{|l|}{$\begin{array}{l}\text { Clase Cyanophyceae } \\
\text { Familia Chroococcaceae }\end{array}$} \\
\hline $\begin{array}{l}\text { Chroococcus minimus } \\
\text { Familia Microcystaceae }\end{array}$ & $\mathrm{x}$ & $x$ & $\mathrm{x}$ & & \\
\hline Microcystis aeruginosa & $x$ & $x$ & $x$ & & \\
\hline $\begin{array}{l}\text { Gloeocapsa sp. } \\
\text { Familia Nostocaceae }\end{array}$ & $\mathrm{x}$ & & & $x$ & $x$ \\
\hline Anabaena sp. & $x$ & $x$ & $x$ & $x$ & \\
\hline \multicolumn{6}{|l|}{ 6. Miozoa } \\
\hline \multicolumn{6}{|l|}{$\begin{array}{l}\text { Clase Dinophyceae } \\
\text { Familia Ceratiaceae }\end{array}$} \\
\hline $\begin{array}{l}\text { Ceratium hirundinella } \\
\text { Familia Peridiniaceae }\end{array}$ & $x$ & $x$ & $x$ & $x$ & \\
\hline Peridinium cintum & & $x$ & & $x$ & \\
\hline \multicolumn{6}{|l|}{ 7. Euglenozoa } \\
\hline \multicolumn{6}{|l|}{$\begin{array}{c}\text { Clase Euglenophyceae } \\
\text { Familia Phacidae }\end{array}$} \\
\hline Phacus tortus & & & $x$ & & \\
\hline
\end{tabular}

En 2018, la diversidad de especies por mes se observa de mayor a menor de la siguiente manera: octubre con quince especies, septiembre con trece especies, diciembre con once especies y noviembre con ocho especies. En 2019, febrero registró siete especies. La flora ficológica del lago presentó una sucesión temporal. Algunas especies fueron dominantes, al registrarse en cinco o en cuatro de los meses de muestreo, como Staurastrum bulbosum, Anabaena sp. y Ceratium hirundinella. Otras fueron especies temporales, como Fragilaria crotonensis, Ephitemia turgida, Asterionella formosa, Eudorina elegans, Chlorococcus minimus, Gleocapsa sp. y Microcystis aeruginosa. Otras especies más fueron esporádicas, ya que estuvieron presentes sólo en dos o un mes de muestreo, como Cocconeis placentula, Cymbella sp., 
Rhopalodia sp., Closterium acutum, Mougeotia sp., Volvox sp., Zygnema sp. y Phacus tortus (tabla 1). En septiembre de 2018 se observó una floración de Anabaena sp., mientras que en febrero de 2019 se presentó una floración de A. formosa. Del total de especies reconocidas se considera a Volvox sp. como un nuevo registro para este lago.

\section{Discusión}

Aunque actualmente el conocimiento en México respecto al fitoplancton es escaso, existe un mayor interés por el conocimiento de estos organismos y se están llevando a cabo diversos estudios para contribuir al reconocimiento y registro de especies nuevas y ya registradas (Novelo y Tavera, 2011). En Morelos, los estudios sobre fitoplancton se han desarrollado principalmente en sistemas acuáticos lóticos, mientras que los estudios en ambientes lénticos son pocos (Mora Navarro et al., 2004).

La diversidad del fitoplancton registrada en el presente trabajo es semejante a la reportada por otros autores para este mismo sitio, como Rodríguez y Tavera (1998) y Rodríguez et al. (2003), pero es menor a la registrada por Godínez Ortega et al. en 2017. A pesar de ello, se considera que el lago que fue objeto de este estudio presenta una diversidad alta en relación con otros lagos de México; además, muestra una relación geográfica afín a las zonas templadas.

Existen variaciones en cuanto a la presencia de especies en el lago, ya que se observan especies dominantes, temporales y esporádicas. Diversos autores señalan que estas variaciones se deben a los cambios en las condiciones abióticas que se presentan a lo largo del año, relacionados principalmente con la temperatura, y el aporte y la remoción de nutrientes tras la temporada de lluvias (Guamán Galarza et al., 2012; Zalocar de Domitrovic et al., 1998). Adicionalmente, las especies aquí reconocidas obedecen en general a una condición de amplia distribución (Coritoma et al., 2018).

Cabe señalar que la clase dominante fue Bacillariophyceae, lo cual coincide con lo mencionado por Oliva Martínez et al. (2014), quienes indican que la clase taxonómica dominante del fitoplancton en México es Bacillariophyceae. En estudios anteriores en este lago se señala a esta clase como la más diversa (Rodríguez et al., 2003).

Respecto a la floración observada de Anabaena sp., ésta se presentó en septiembre, que es temporada de lluvias, por lo que se atribuye la presencia de esta especie a las condiciones de esa época: se incrementa la cantidad de nutrientes como consecuencia del arrastre desde las laderas de las montañas hacia el lago y por el mayor movimiento de la columna de agua, lo que origina un flujo de los sedimentos con aporte de nutrientes del fondo, que produce una proliferación algal en el cuerpo de agua (Morales et al., 2016). Sin embargo, no se observó ninguna alteración con respecto a otros organismos ocasionada por dicho florecimiento.

Año 16, núm. 40, noviembre 2020-marzo 2021 
Otra floración se presentó en el mes de febrero de 2019, la de A. Formosa. En investigaciones anteriores se reconoció esta especie como dominante por su abundancia y por presentarse en todas las épocas del año. Si bien para este estudio la floración de $A$. Formosa fue observada en tres de los cinco meses, sólo pudo apreciarse una abundancia considerable en el mes antes mencionado, lo que sugiere que se deben estar presentando cambios en el sistema que no favorecen su presencia (Rakaj, 2010). Por su parte, la especie con mayor número de registros fue Staurastrum bulbosum, la cual fue reportada en 2017 por Godínez Ortega et al. como nueva especie en México. La dominancia de dicha especie probablemente se debe a que puede soportar cambios en las condiciones ambientales que otras especies no.

De manera general, podríamos considerar al lago Zempoala como un sistema léntico estable con ciertas condiciones de eutrofia debido a la diversidad registrada, así como a los florecimientos de las dos especies antes mencionadas. Se concluye, con los resultados aquí presentados, que la diversidad del fitoplancton en el lago presenta poca variación con respecto a los estudios previos realizados en este sitio y que la composición algal presenta una distribución cosmopolita, sin dejar de lado el nuevo registro de Volvox sp.

\section{Figura 1}

\section{Bacillariophyceae: a) Asterionella formosa, b) Cocconeis placentulla, c) Cymbella sp., d) Fragillaria crotonensis, e) Ephitemia turgida, f) Ephitemia sorex, g) Rhopalodia sp., h) Gomphonema acuminatum}
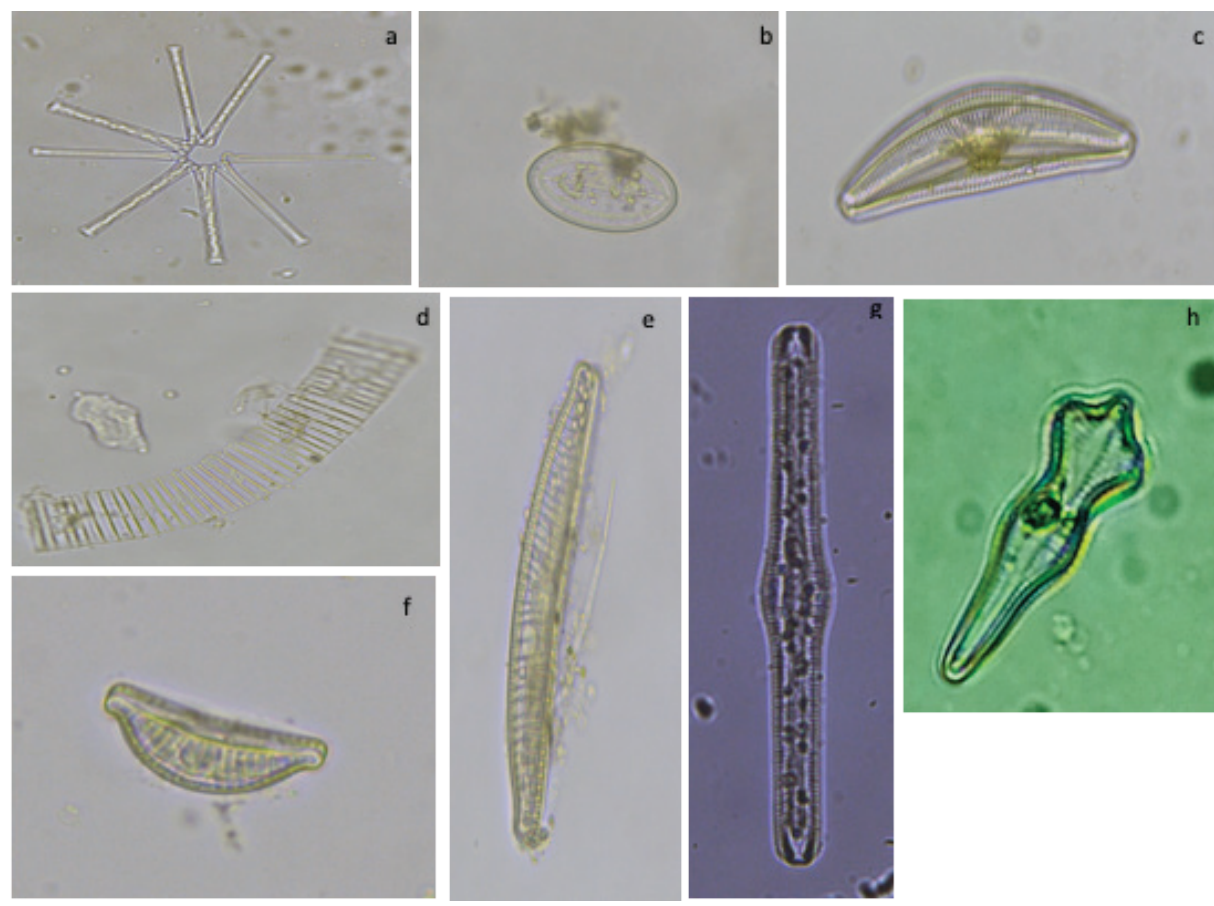

Año 16, núm. 40, noviembre 2020-marzo 2021 


\section{Figura 2}

Charophyceae: a) Closterium acutum, b) Staurastrum bulbosum, c) Mougeotia sp., d) Cosmarium subtumidum, e) Zygnema sp.
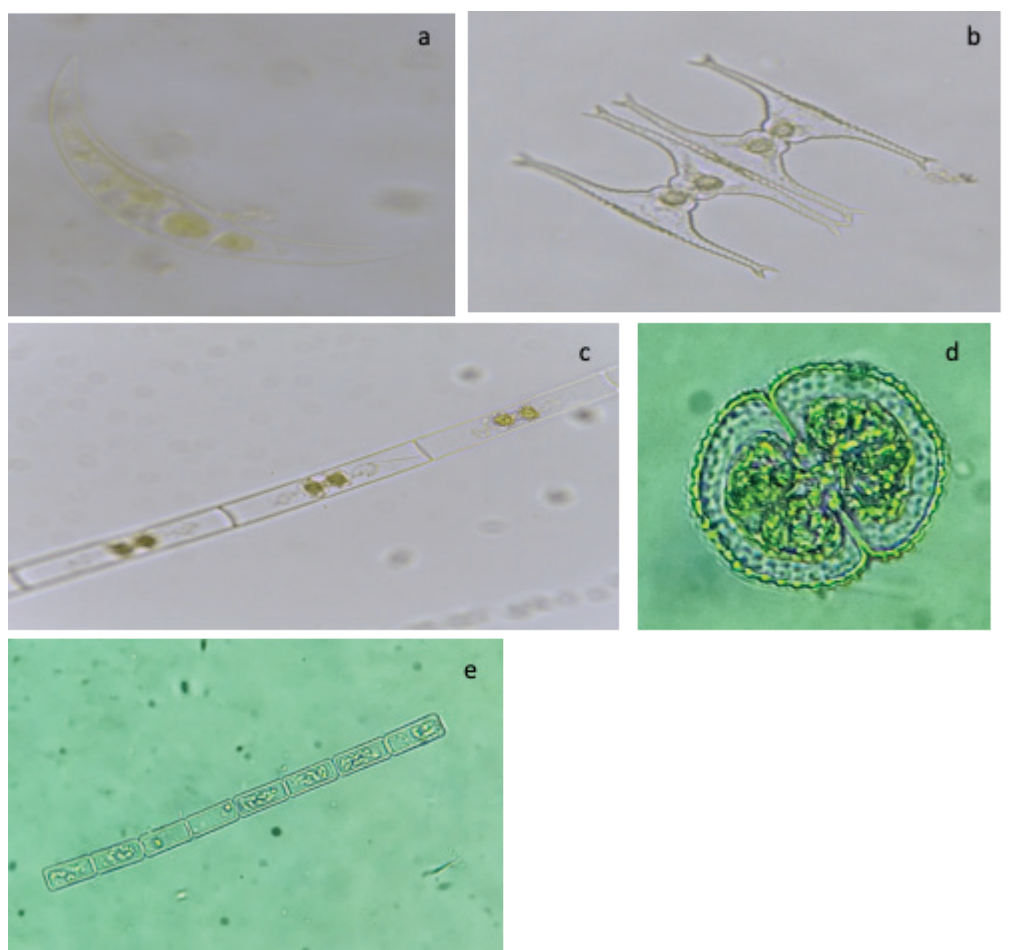

Figura 3

Chlorophyceae: a) Scenedesmus quadricauda, b) Radiofilum conjunctivum, c) Volvox sp., d) Eudorina elegans, e) Stauridium tetras
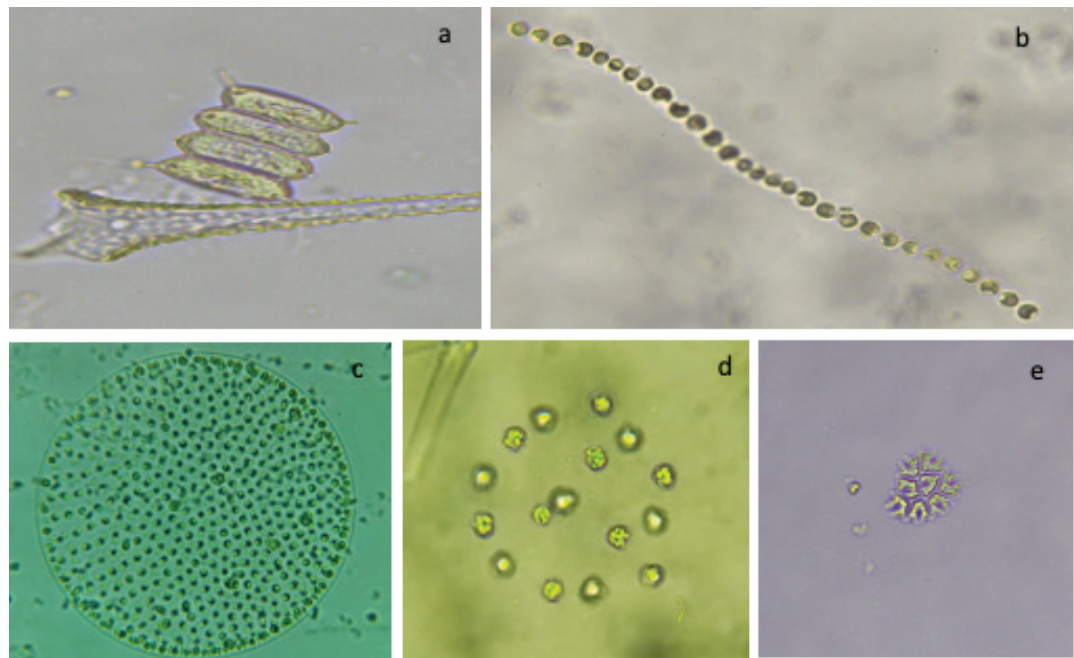

Año 16, núm. 40, noviembre 2020-marzo 2021

ISSN: 2007-1760 (impreso) 2448-9026 (digital) | DOI: 10.30973/inventio/2021.16.40/4 


\section{Figura 4}

Cyanophyeae: a) Mycrocystis aeruginus, b) Gloeocapsa sp.,

c) Anabaena sp., Dinophyceae d) Peridinium cinctum,

e) Ceratium hirundinella, Euglenophyceae, f) Phacus tortus

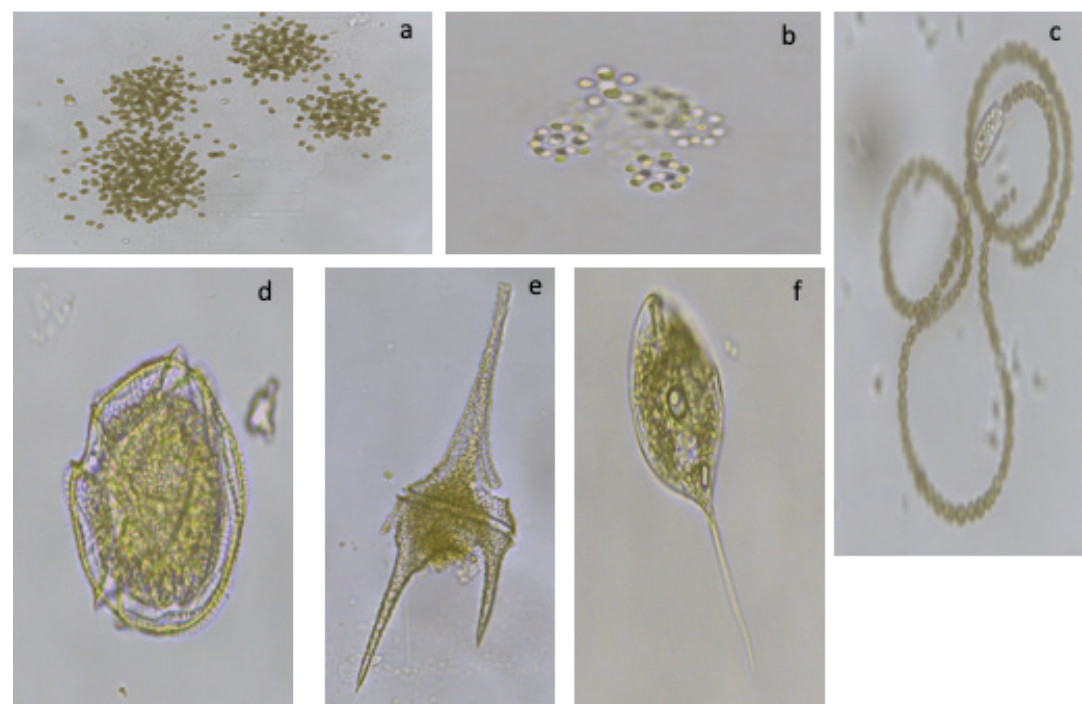

\section{Referencias}

Coritoma, M. B., Castro, K. R., Sánchez, T. L., Ugaz, L. T., Peña, E. J., Prada, D. M., Sevillano, A. S. y Rubina, A. D. (2018). Evaluación de la diversidad de algas fitoplanctónicas como indicadores de la calidad del agua en lagunas altoandinas del departamento de Pasco (Perú). Ecología Aplicada, 17 (1), 119-132. https://doi.org/10.21704/rea.v17i1.1180

E. Graham, L., M. Graham, J., E Cook M. y W. Wilcox, L. (2016). Algae. (3. . ed.). Pearson Benjamin Cummings.

Godínez Ortega, J. L., Oliva Martínez, M. G., Escobar Oliva, M. A. y Mendoza Garfias, M. B. (2017). Diversidad algal del Parque Nacional Lagunas de Zempoala, México, excepto diatomeas, Hidrobiológica, 27 (1), 45-58. http://www.scielo.org.mx/scielo. php?script=sci arttext\&pid=S0188-88972017000100045\&lng=es\&tlng=es

Guamán Galarza, A. M., Velásquez Empuño A. E. y Jaramillo Minaya, M. A. (2012). Aplicación de la cianobacteria Anabaena sp.cpb 4337 como bioindicador de toxicidad por metales pesados en el embalse ESPOL. [Tesis de Grado, Escuela Superior Politécnica del Litoral]. http://www.dspace.espol.edu.ec/xmlui/handle/123456789/20889

G. Wetzel, R. (2001). Limnology: lake and river ecosystems. (3a ed.). Academic Press.

Guiry, M. D. y Guiry, G. M. (2020). AlgaeBase. Publicación electrónica mundial, Universidad Nacional de Irlanda, Galway. http://www.algaebase.org 
Mora Navarro, M. R., Vázquez García, J. A. y Vargas Rodríguez, Y. L. (2004). Ordenación de comunidades de fitoplancton en el lago de Chapala, Jalisco-Michoacán, México. Hidrobiológica, 14 (2), 91-103. http://www.scielo.org.mx/scielo.php?script=sci arttex $\underline{\text { t\&pid }=S 0188-88972004000200002}$

Morales, A. P., Ortiz, A. O., Quijano-Scheggia, S. I., Espinosa Rodríguez, C. A. y Jiménez Santos, M. A. (2016). Estado actual del estudio de cianobacterias dulceacuícolas formadoras de florecimientos en el centro de México. En García-Mendoza, E., Quijano-Scheggia, S. I., Olivos-Ortiz, A. y Núñez-Vázquez, E. J. (eds.), Florecimientos Algales Nocivos en México (pp. 408-421). Centro de Investigación Científica y de Educación Superior de Ensenada, Baja California.

Novelo, E. y Tavera, R. (2011). Un panorama gráfico de las algas de agua dulce de México. Hidrobiológica, 21 (3), 333-341. https://www.researchgate.net/publication/236176066 Un panorama grafico de las algas de agua dulce de Mexico

Oliva Martínez, M. G., Godínez Ortega, J. L. y Zuñiga Ramos, C. A. (2014). Biodiversidad del fitoplancton de aguas continentales en México. Revista Mexicana de Biodiversidad, 85 (12), 54-61. https://doi.org/10.7550/rmb.32706

Ortega, M. (1984). Catálogo de algas continentales recientes de México. Instituto de Biología, Universidad Nacional Autónoma de México.

Rakaj, M. (2010). Biological Water Quality of Lake Shkodra Based on the Diatoms and cyanobacteria Bioindicator Species. BALWOIS, 25, 3-6. http://balwois.com/wp-content/ uploads/old proc/ffp-1725.pdf

Rodríguez, J. G. y Tavera, R. (1998). Fitoplancton del Lago Zempoala. Boletín de la Sociedad Botánica de México, 63, 85-100. https://www.botanicalsciences.com.mx/index.php/ botanicalSciences/article/view/1570/1221

Rodríguez, J. G., Molina Astudillo, F. I., Castelán, H. Q. y Albarrán, R. T. (2003). Especies del fitoplancton en el lago Tonatiahua, Morelos, México. Acta Universitaria, 13 (2), 53-66. https://doi.org/10.15174/au.2003.270

Rodríguez, J. G., Molina Astudillo, F. I., Vargas, M. D. y Castelán, H. Q. (2010). Componentes fitoplanctónicos y zoobentónicos en el Lago Zempoala, Morelos, México, Acta Universitaria, 20 (2), 23-30. https://doi.org/10.15174/au.2010.75

Zalocar de Domitrovic, Y., Asselborn, V. y Casco, S. (1998). Variaciones espaciales y temporales del fitoplancton en un lago subtropical de Argentina. Revista Brasileira de Biologia, 58 (3), 359-382. https://doi.org/10.1590/S0034-71081998000300003 